\title{
Incidência de fibro edema geloide em mulheres caucasianas jovens
}

Incidence of cellulite in young Caucasian women

Aline Fernanda Perez Machado', Rogério Eduardo Tacani', Juliano Schwartz', Richard Eloin Liebano', Juliane de Lemos Armada Ramos', Thiago Frare'

\begin{abstract}
Resumo
0 fibro edema geloide é uma alteração esteticamente indesejável que acomete mulheres em alguma época da vida. De acordo com a classificação proposta por Ulrich, existem três graus de fibro edema geloide: I, II e III. 0 objetivo deste estudo foi verificar a incidência da localização corporal e do grau do fibro edema geloide e analisar a porcentagem de gordura em mulheres caucasianas jovens. Foram selecionadas 30 mulheres com média de idade de 20 anos e queixa de fibro edema geloide. As voluntárias foram submetidas à avaliação composta de anamnese, exame físico, avaliação antropométrica e da composição corporal. A média da porcentagem de gordura foi de 27,18 . Portanto, concluiu-se que as regiões glútea e posterior da coxa foram as mais acometidas, o grau I foi o mais incidente, e a média de porcentagem de gordura se mostrou elevada nesta população com queixa de fibro edema geloide.
\end{abstract}

Palavras-chave: Celulite; adiposidade; fisioterapia (especialidades); saúde da mulher; composição corporal.

\begin{abstract}
Cellulite is an unacceptable aesthetic problem, which affects females in some period of life. According to Ulrich's classification proposed there are three cellulite's stages: I, II and III. This study aimed at verifying the incidence of cellulite, by the localization and stage, and to analyze fat percentage in young Caucasian women. Thirty women, who are volunteers, with mean age of 20 years old and complaining of cellulite, were selected. Volunteers were submitted to an evaluation that consisted of anamnesis, physical exam, anthropometric measures and body composition. The fat percentage mean was 27.18. Therefore, it was concluded that cellulite has more incidence in buttocks and posterior thigh, stage I was the most affected, and the fat percentage mean was higher in this population with cellulite's complaints.
\end{abstract}

Keywords: Cellulite; adiposity; physical therapy (specialty); women's health; body composition.

Recebido: 30/09/2008

Revisado: 20/04/2009

Aprovado: 04/05/2009 


\section{Introdução}

O fibro edema geloide (FEG), popularmente conhecido como “celulite”, é uma alteração comum da topografia da pele, indesejável esteticamente, que acomete milhões de mulheres no mundo ${ }^{1-3}$. Este se manifesta por contornos irregulares na pele a partir da puberdade ${ }^{1,2,4-6}$. É definido como uma disfunção metabólica localizada, do tecido subcutâneo e da derme, a qual provoca alteração na forma corporal feminina, causada pelo excesso de tecido adiposo retido no septo fibroso ${ }^{1,5}$ e por projeções deste na derme².

O termo celulite foi descrito pela primeira vez, na França, por Alquier e Paviot, por volta de $1920^{1,4,7}$, estes acreditavam se tratar de uma distrofia celular complexa não inflamatória de tecido mesenquimal causada por disfunção no metabolismo de água, como resposta a qualquer tipo de agressão (traumática, tóxica ou endócrina), produzindo saturação do tecido adjacente por líquido intersticial, caracterizando retenção deste ${ }^{1,2,6,7}$. Em 1928, Lagèse identificou presença de soro no espaço intersticial, fibrose e retração esclerótica, caracterizando a inadequação do termo celulite, conforme descrito por Pires de Campos 8 .

Outras nomenclaturas são utilizadas para designar a condição “celulite estética”, como: lipodistrofia ginoide (proposta pelos célebres professores Ciporkin e Paschoal ${ }^{7}$ ), lipoesclerose nodular, paniculopatia edemato-fibroesclerótica, paniculose, dermoipodermose, dermatopaniculopatia edematosa fibrosa e esclerosa, entre outros ${ }^{1,2,4,6,8}$.

Atualmente, diversas hipóteses sugerem que a fisiopatologia do FEG ocorra pela hiperpolimerização anormal do tecido conectivo associada a alterações primárias do tecido adiposo e alterações microcirculatórias ${ }^{1,2,9}$.

De acordo com a classificação proposta por Ulrich ${ }^{10}$, existem três graus de FEG. O grau I ou brando ocorre quando as alterações cutâneas são percebidas somente durante a compressão dos tecidos (teste de casca de laranja ou contração muscular) geralmente na posição ortostática, pois quando a pessoa está deitada frequentemente não se percebe sua presença. O grau II ou moderado apresenta as alterações cutâneas de forma espontânea, sem compressão dos tecidos, pode ser visível com a voluntária deitada e com as margens bem delimitadas. O grau III ou grave apresenta alterações cutâneas tanto em posição deitada ou em ortostatismo, ficando com a pele enrugada e flácida adquirindo a aparência do chamado saco de nozes.

É descrito como uma alteração da superfície da pele com aspecto acolchoado, em casca de laranja ou em saco de nozes ${ }^{1,3-6,9,11}$, que pode acarretar em sérias complicações levando a problemas que interferem na aparência física e alteração da autoimagem; perturbações orgânicas, quadros álgicos nas zonas acometidas, diminuição das atividades funcionais, e em casos mais graves pode levar até quase a imobilidade dos membros inferiores ${ }^{12,13}$.

Sua etiologia é considerada multifatorial, pois neste processo existem vários fatores envolvidos, como os desencadeantes, os pre- disponentes e os agravantes ${ }^{1,5,6,14}$. O hiperestrogenismo é considerado como principal fator desencadeante ${ }^{7}$. Os fatores predisponentes são de origens genética, sexual, étnica, pelo biotipo corporal, pela distribuição de tecido adiposo e pelos receptores envolvidos. E dentre os agravantes estão os hábitos alimentares inadequados, sedentarismo, estresse, medicamentos e gravidez 6 .

Segundo a escassa literatura, é uma afecção de alta prevalência, atingindo todas as raças, sendo mais comum nas caucasianas do que nas africanas ${ }^{4,13}$, mas com poucos estudos envolvendo o tema. As áreas corporais acometidas são a região pélvica, os membros inferiores e o abdome ${ }^{1}$, podendo ser afetadas também a região das mamas; a região superior dos braços e da nuca, as quais apresentam um padrão feminino de deposição de tecido adiposo; porém, a região súpero-posterior e lateral das coxas e a região glútea são as áreas mais suscetíveis ao desenvolvimento desta condição ${ }^{4}$.

Apesar disto, não são conhecidos estudos que informam a incidência do FEG por região corporal quanto ao seu grau de evolução e à simetria do acometimento. É uma entidade clínica independente da obesidade, porém as pacientes podem apresentar alteração da massa $\operatorname{adiposa}^{15}$.

Portanto, o objetivo deste trabalho foi verificar a incidência da localização corporal e do grau do FEG e analisar a porcentagem de gordura em mulheres caucasianas jovens.

\section{Casuística e métodos}

Foram selecionadas 30 voluntárias com idades entre 18 e 29 anos e queixa de FEG.

O projeto do estudo foi aprovado pela Comitê de Ética em Pesquisa da Universidade Cidade de São Paulo (Unicid), em São Paulo. Todas as voluntárias assinaram e concordaram com os itens especificados no termo de consentimento livre e esclarecido.

Os critérios de inclusão foram: mulheres caucasianas, ativas não treinadas, com queixa de FEG, índice de massa corporal (IMC) entre 18,5 e $29,9 \mathrm{~kg} / \mathrm{m}^{2}$, classificado entre adequado $\left(18,5-24,9 \mathrm{~kg} / \mathrm{m}^{2}\right)$ e sobrepeso $\left(25-29,9 \mathrm{~kg} / \mathrm{m}^{2}\right)$, segundo a Organização Mundial da Saúde ${ }^{16}$, adaptadas em meio aquático e com ausência de patologias associadas.

Os critérios de exclusão foram: mulheres em tratamento farmacológico ou clínico para emagrecimento ou estético, prática regular de atividade física, período pós-operatório menor que seis meses, gravidez e lactação.

As voluntárias foram submetidas a uma avaliação composta de anamnese; exame físico, por meio de inspeção e palpação; avaliação antropométrica e da composição corporal pelo método de pesagem hidrostática.

$\mathrm{Na}$ anamnese, foram coletados os dados demográficos, hábitos de vida e antecedentes ginecológicos das voluntárias. No exame físico, foram observadas as características das adiposidades localizadas e avaliação do FEG. 
As adiposidades localizadas foram classificadas quanto à consistência, flácida ou compacta, e quanto ao biotipo, sendo consideradas as formas androide, ginoide ou mista ${ }^{12}$.

O grau do FEG foi determinado por meio da classificação proposta por Ulrich ${ }^{10}$. Quanto à localização, avaliaram-se os lados direito e esquerdo e foram divididos os segmentos corporais em glúteo; região posterior, anterior, medial e lateral de coxa.

Durante a inspeção, analisou-se a presença de irregularidades e/ou ondulações na superfície da pele, principalmente aspectos acolchoados, de casca de laranja ou de “saco de nozes”. A palpação foi feita por pinçamento e compressão tecidual, realizados pelos testes de preensão e de casca de laranja ${ }^{7}$.

A avaliação antropométrica foi feita por meio da aferição da estatura e da massa corporal. A massa corporal foi aferida por meio de uma balança da marca Welmy ${ }^{\circledR}$, com carga máxima de $150 \mathrm{~kg}$ e divisões de $100 \mathrm{~g}$. A estatura foi avaliada no estadiômetro da marca Sanny ${ }^{\circledR}$, que possui comprimento máximo de $2 \mathrm{~m}$ e divisões de $1 \mathrm{~mm}$. As voluntárias permaneceram com seus pés encostados na parede durante a medição. Para quantificar a estatura e massa corporal das voluntárias, elas deveriam ficar na posição ortostática, trajar roupas de banho com duas peças, cabeça em linha reta, membros superiores ao longo do corpo e pés descalços e paralelos à linha dos ombros.

Para a avaliação da composição corporal, utilizou-se o método indireto de pesagem hidrostática, assim determinou-se a porcentagem de gordura $(\% \mathrm{G})$, peso da massa gorda (PMG) e peso da massa magra $(\mathrm{PMM})^{17}$. Esta técnica foi realizada em um tanque apropriado, localizado no Laboratório de Pesquisa em Educação Física e Fisioterapia (LAPEFI II) da Unicid (Figura 1).

Para a execução deste exame, cada voluntária permanecia com os pés descalços, trajando roupas de banho apropriadas e touca de piscina, para manutenção da higiene do tanque, sentada numa gaiola suspensa por uma corrente unida a uma balança digital com precisão de $5 \mathrm{~g}$ devidamente calibrada.

Cada voluntária foi orientada a realizar uma expiração forçada máxima, em seguida realizar uma flexão de tronco, e manter-se em apneia em torno de cinco segundos para estabilização dos valores da balança (Figura 2). Foram realizadas aproximadamente oito mensurações submersas, sendo que ao final era realizada uma média com as três melhores medidas ${ }^{17,18}$.

O peso hidrostático foi transformado em densidade corporal utilizando a seguinte equação ${ }^{19}: \mathrm{DC}(\mathrm{g} / \mathrm{mL})=\mathrm{PS} /[(\mathrm{PS}-\mathrm{PA}) / \mathrm{DA}]-$ $(\mathrm{VR}+\mathrm{VGI})$, onde: $\mathrm{DC}=$ densidade corporal; PS = massa corporal em solo $(\mathrm{kg}) ; \mathrm{PA}=$ peso corporal na água $(\mathrm{kg}) ; \mathrm{DA}=$ densidade da água; $\mathrm{VR}=$ volume residual $(\mathrm{L})$ e VGI = volume de gás gastrointestinal (constante $100 \mathrm{~mL}=0,1 \mathrm{~L}$ ). Para tanto, era necessária a determinação do $\mathrm{VR}^{19}: \mathrm{VR}=0,021$ (idade em anos) $+0,023$ (estatura em $\left.\mathrm{cm}\right)-2,978$. Assim, pode-se estimar a $\% \mathrm{G}^{19}: \% \mathrm{G}=(495 / \mathrm{DC})-450$. E, por fim, determinou-se o PMG e o $\mathrm{PMM}^{19}$ : $\mathrm{PMG}=\mathrm{MC}(\mathrm{kg}) \times \mathrm{\%}$ e $\mathrm{PMM}=$ MC (kg) - PMG, onde MC = massa corporal.

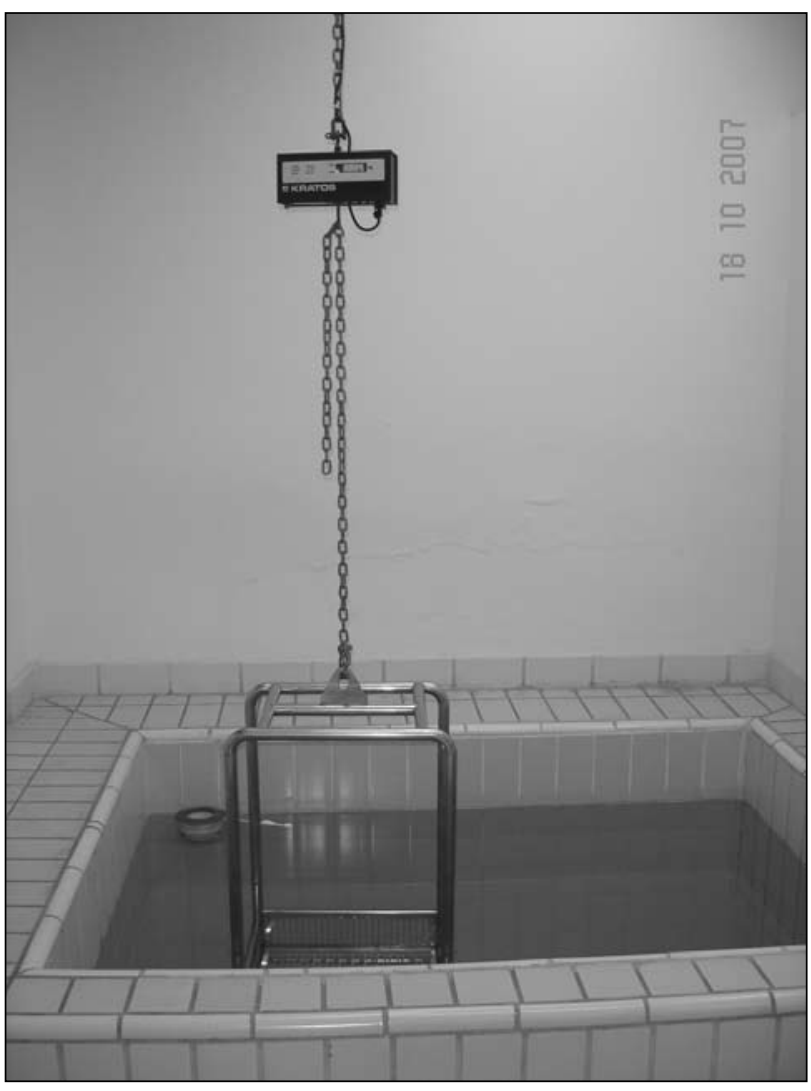

Figura 1 - Tanque para pesagem hidrostática

\section{Análise estatística}

A normalidade dos dados foi verificada com o teste de Shapiro-Wilk. A estatística descritiva constou do cálculo de média e desvio padrão.

Para verificar a associação entre as variáveis, utilizou-se a correlação de Spearman. Foi considerado: $|0,00|$ a $|0,19|$ - correlação bem fraca; $|0,20|$ a $|0,39|$ - correlação fraca; $|0,40|$ a $|0,69|$ - correlação moderada; $|0,70|$ a $|0,89|$ - correlação forte e $|0,90|$ a $|1,00|$ - correlação muito forte, representada pela letra " $r$ ".

Foi assumido valor de $\alpha \leq 5 \%$ ( $p \leq 0,05)$ como estatisticamente significante. Para as análises foram utilizados os softwares estatísticos Prism 5.0 for Windows (GraphPad Software, Inc.) e SPSS Statistics 17.0 for Windows (SPSS, Inc.).

\section{Resultados}

Foram avaliadas 30 mulheres caucasianas, ativas, não treinadas, com média de idade de 20,37 anos $( \pm 1,65)$ e queixa de FEG. Quanto aos hábitos de vida, 3,3\% $(n=1)$ alegaram ser tabagistas, 53,3\% $(n=16)$ referiram uso de anticoncepcional hormonal e não havia etilista.

A média da massa corporal foi de $59,63 \mathrm{~kg}( \pm 7,60)$ e da estatura foi de $161,3 \mathrm{~cm}( \pm 5,09)$, apresentando IMC médio de $22,95 \mathrm{~kg} / \mathrm{m}^{2}$ $( \pm 2,92)$, classificado como adequado ${ }^{16}$. Destas, $66,7 \%(n=20)$ apre- 


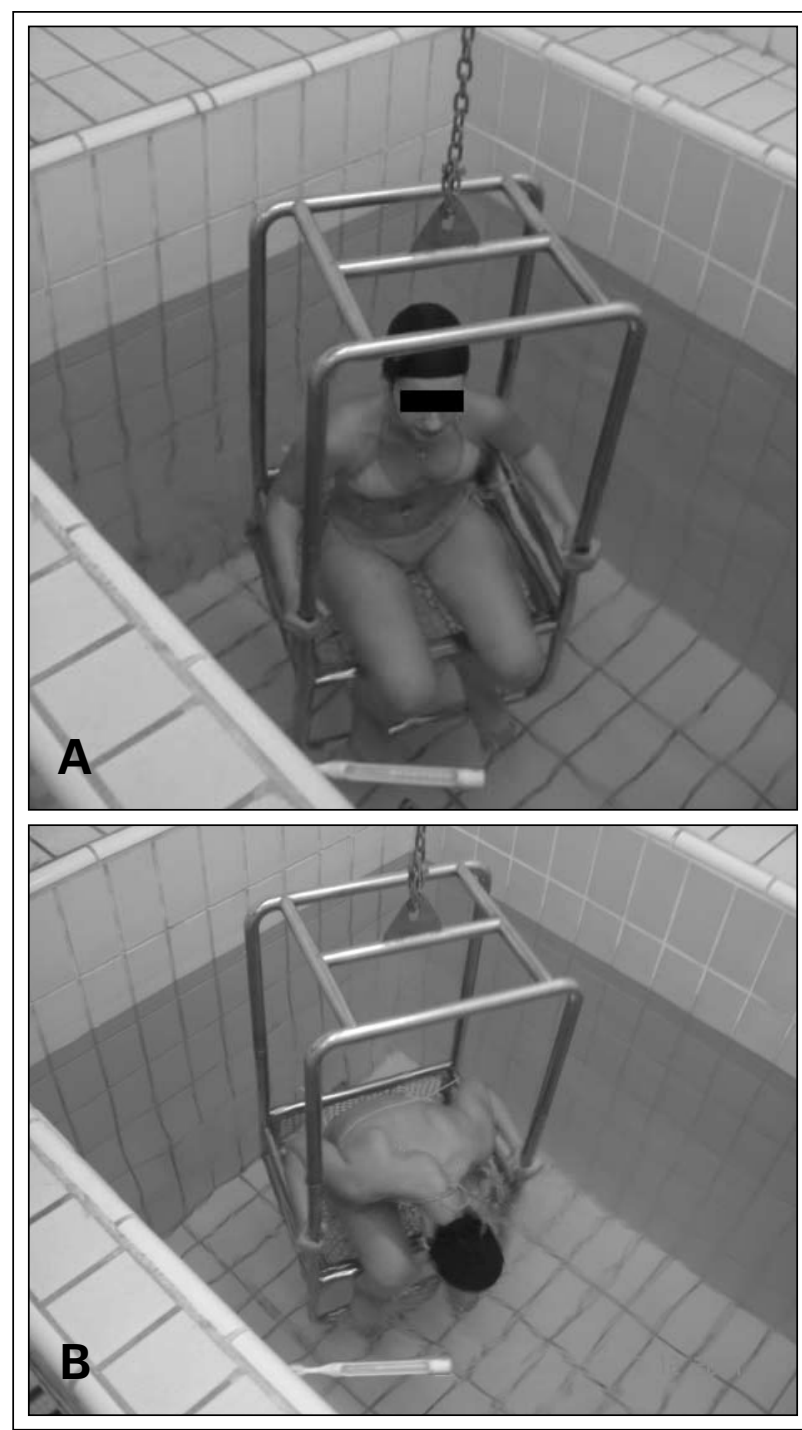

Figura 2 - Realização do procedimento; (A) posição inicial; (B) flexão de tronco e apneia por cinco segundos

sentavam IMC médio de $20,4 \mathrm{~kg} / \mathrm{m}^{2}$ ( $\left.\pm 0,94\right)$, considerado adequado ${ }^{16}$; e $33,3 \%(n=10)$ apresentavam IMC médio de $26,6 \mathrm{~kg} / \mathrm{m}^{2}( \pm 1,08)$, considerado sobrepeso ${ }^{16}$.

Na pesagem hidrostática, a média da \%G corporal foi de 27,18 $( \pm 7,75)$, do PMG foi de $16,45 \mathrm{~kg}( \pm 4,83)$ e do PMM de $43,17 \mathrm{~kg}$ $( \pm 6,18)$.

No exame físico foi observada a presença de adiposidades localizadas, de consistência flácida em 96,6\% ( $n=29)$ e compacta em 3,3\%

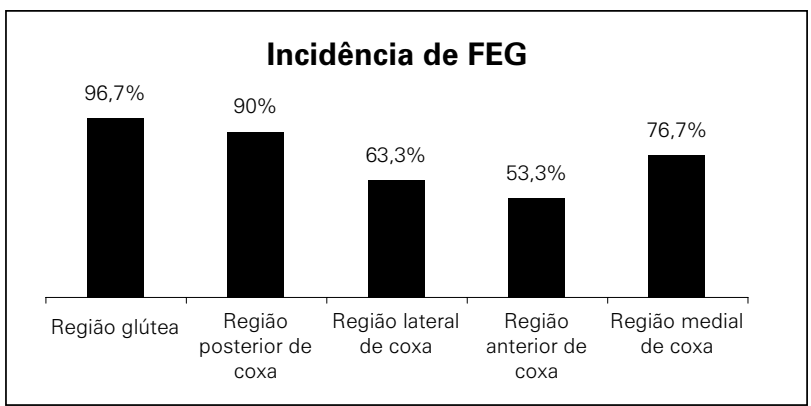

Figura 3 - Incidência de FEG entre as regiões glútea, posterior, lateral, anterior e medial de coxa

$(\mathrm{n}=1)$. Em relação ao biotipo, $80 \%(\mathrm{n}=24)$ eram ginoides, $3,3 \%$ $(n=1)$ androides e 16,6\% ( $n=5)$ mistas.

Quanto à presença de FEG, observou-se que maioria delas apresentou FEG em mais de um local avaliado, com graus variados e tendência à simetria.

A Figura 3 demonstra a incidência de FEG nas diferentes regiões corporais avaliadas. As mais incidentes foram as regiões glútea $(96,7 \%)$ e posterior da coxa (90\%), seguida da região medial da coxa (76,7\%), depois a região lateral da coxa $(63,3 \%)$, e por último a região anterior da coxa $(53,3 \%)$.

A Tabela 1 demonstra a correlação entre a localização e o grau do FEG. Para o cálculo das correlações, os lados direito e esquerdo da variável grau do FEG das regiões glútea, posterior e anterior de coxa foram unificados em uma única variável. Quando os lados apresentavam graus diferentes entre si, foi adotado o maior grau entre os lados para a realização de teste estatístico.

$\mathrm{Na}$ região glútea, bilateralmente foi observada a presença de FEG em $96,7 \%(\mathrm{n}=29)$ das voluntárias, destas $20 \%(\mathrm{n}=6)$ apresentaram o grau I, $40 \%(n=12)$ o grau II, $36,7 \%(n=11)$ o grau III, destas $10 \%$ $(n=3)$ apresentaram graus diferentes entre os glúteos direito e esquerdo, e 3,3\% ( $n=1)$ não apresentaram FEG nesta região.

$\mathrm{Na}$ região posterior da coxa, bilateralmente o FEG esteve presente em $90 \%(n=27)$ das voluntárias, assim em $30 \%(n=9)$ foi observado o grau I, em $50 \%(n=15)$ o grau II, em 10\% ( $n=3)$ o grau III, e destas $3,3 \%(n=1)$ tinham graus diferentes entre os lados, e $10 \%(n=3)$ não apresentaram a afecção ao exame.

A região medial denotou mais acometimento que a lateral, sendo os porcentuais de $76,6 \%(\mathrm{n}=23)$ e $63,3 \%(\mathrm{n}=19)$ para cada uma, respectivamente. Na região medial, o grau I apresentou-se em $66,7 \%$

Tabela 1 - Correlação entre a localização e o grau do FEG

\begin{tabular}{lcccc}
\hline \multirow{2}{*}{ Localização } & \multicolumn{3}{c}{ Grau } \\
\cline { 2 - 5 } & Grau I n (\%) & Grau II n (\%) & Grau III n (\%) & Não apresenta n (\%) \\
\hline Região glútea & $6(20)$ & $12(40)$ & $11(36,7)$ & $1(3,3)$ \\
Região posterior de coxa & $9(30)$ & $15(50)$ & $3(10)$ & $3(10)$ \\
Região lateral de coxa & $6(20)$ & $13(43,3)$ & - & $11(36,7)$ \\
Região anterior de coxa & $15(50)$ & $1(3,3)$ & - & $14(46,7)$ \\
Região medial de coxa & $20(66,7)$ & $3(10)$ & - & $7(23,3)$ \\
\hline
\end{tabular}


$(n=20)$, o grau II em 10\% ( $n=3)$ e 23,3\% ( $n=7)$ não apresentaram algum grau. Já na região lateral, 20\% $(n=6)$ tinham grau I, 43,3\% (n= 13) grau II e 36,7\% (n= 11) não estavam acometidas.

A presença do FEG bilateralmente na região anterior da coxa representou 53,3\% $(n=16)$, com grau I correspondendo a $50 \%(n=15)$, o grau II a 3,3\% (n=1) e 46,7\% (n=14) não apresentaram o FEG.

Após a correlação dos dados, observou-se que não houve correlação significativa dos graus do FEG entre a \%G corporal e PMM separadamente. Porém, houve uma correlação significativa positiva do grau do FEG entre o PMG na região glútea $(r=0,486 ; \mathrm{p}=0,007)$ e na região posterior da coxa $(r=0,490 ; p=0,009)$, também em relação ao grau do FEG entre a massa corporal $(r=0,473 ; p=0,010)$ e o IMC $(\mathrm{r}=0,479, \mathrm{p}=0,009)$ na região glútea, classificadas como uma correlação moderada.

Portanto, pode-se dizer que o grau do FEG na região glútea parece ter associação diretamente proporcional com as variáveis PMG, massa corporal e IMC; e na região posterior parece ter associação diretamente proporcional com a variável PMG.

\section{Discussão}

Neste estudo preconizou-se a utilização da pesagem hidrostática, por ser um método de avaliação da composição corporal considerado como padrão-ouro ${ }^{19}$.

Apesar da amostra se enquadrar em padrões adequados de $\mathrm{IMC}^{16}$, a média de \%G verificada foi de 27,18 $( \pm 7,75)$. Apenas $20 \%(n=6)$ apresentavam até $25 \% \mathrm{G}$; assim, $80 \%(\mathrm{n}=24)$ estavam acima do preconizado, pois de acordo com a classificação proposta por Pitanga ${ }^{20}$, mulheres com valores acima de $25 \% \mathrm{G}$ são consideradas elevadas.

Mesmo aquelas mulheres que possuem IMC considerado adequa$\mathrm{do}^{16}$ podem apresentar gordura corporal aumentada, pois este índice não analisa individualmente os compartimentos de massa magra e de gordura $^{15}$. Analisando separadamente os dados referentes ao IMC adequado e sobrepeso, $33,3 \%(\mathrm{n}=10)$ foram classificadas com sobrepeso, com IMC médio de $26,6 \mathrm{~kg} / \mathrm{m}^{2}( \pm 1,08)$; e $66,7 \%(\mathrm{n}=20)$ que foram classificadas como adequado tinham IMC médio de $20,4 \mathrm{~kg} / \mathrm{m}^{2}$ ( \pm 0,94), e os \%G corporal destes grupos foram de 30,1 ( \pm 4,63) e 26,5 ( \pm $7,58)$, respectivamente, não demonstrando diferenças quanto ao IMC no que se refere ao aumento da $\% \mathrm{G}$, pois ambos pontuaram $>25 \% \mathrm{G}$.

Neto, Franscischelli e Oliveira ${ }^{15}$ analisaram a composição corporal por impedância bioelétrica de 77 mulheres caucasianas (idade média de 30,7 anos), com graus variados de FEG. Os autores verificaram que, mesmo as pacientes com IMC $<25 \mathrm{~kg} / \mathrm{m}^{2}$ apresentavam $\% \mathrm{G}$ corporal aumentada, demonstrando a associação entre o FEG e o excesso de gordura corporal total, embora apresentassem peso adequado ou mesmo abaixo do considerado adequado.

As mulheres com IMC classificado adequado ${ }^{16}$ apresentaram gordura corporal aumentada. Das 30 mulheres avaliadas neste estudo, 73,7\% $(n=14)$ apresentaram \% considerada elevada. Já, con- siderando as mulheres na faixa de sobrepeso, $90 \%(n=10)$ apresentaram $>25 \% \mathrm{G}^{20}$.

Como é possível observar, tanto nas mulheres com peso adequado quanto com sobrepeso, há uma predominância de mulheres com \%G considerada elevada, sendo esta predominância mais expressiva no grupo de mulheres classificadas em sobrepeso.

Todas as avaliadas apresentaram adiposidades localizadas, $80 \%$ eram ginoides (16,6\% mistas e apenas 3,4\% androides), biotipo que apresenta deposição regional excessiva de gordura com alterações nas regiões alvo principalmente de quadris e coxas, conforme a herança genética associada a alterações hormonais ${ }^{7}$.

Embora este tipo de adiposidade não tenha consequência negativa na expectativa de vida, pode estar envolvido com a aparência inestética de acordo com os padrões atuais de harmonia corporal ${ }^{15}$.

Apesar de ser considerada uma entidade clínica autônoma e independente da obesidade, as portadoras de FEG podem apresentar gordura corporal total ou regional aumentada, e isto influencia o processo de sua etiopatogenia ${ }^{15}$. Querleux et al. ${ }^{21}$ analisaram imagens por ressonância magnética nuclear (RMN) de mulheres com IMC adequado e FEG visível com ou sem compressão; verificaram um aumento significativo da espessura do tecido adiposo subcutâneo, de duas vezes na camada areolar e seis na camada lamelar, em relação a homens com IMC similar e a mulheres sem FEG e com IMC em torno de $18 \mathrm{~kg} / \mathrm{m}^{2}$.

Mirrashed et al. ${ }^{22}$ também utilizaram análise de microimagens por RMN da derme e do tecido adiposo subcutâneo de 12 mulheres com idade entre 18 e 46 anos, com IMC adequado e obesidade $\left(>30 \mathrm{~kg} / \mathrm{m}^{2}\right)$ com baixo e alto grau de FEG, classificado em quatro graus. Os autores também verificaram que o tecido adiposo subcutâneo de mulheres com alto grau de FEG é mais espesso, apresentando lóbulos adiposos maiores, independentemente do IMC ser aumentado ou adequado.

Ortonne et al. ${ }^{23}$ analisaram a espessura do tecido adiposo subcutâneo por ultrassonografia (10 MHz) de 94 mulheres saudáveis, divididas em três grupos etários (21-30 anos; 31-40 anos; 51-60 anos) subdivididos em presença de FEG grau 2 e grau 0 . Observou-se 30\% de aumento da espessura da dobra cutânea das mulheres com FEG grau 2 em todos os grupos etários.

Segundo Rosenbaum, Prieto, Hellmer et al. ${ }^{24}$, as mulheres com FEG apresentam herniações dos adipócitos da camada areolar na derme, contribuindo para as irregularidades cutâneas.

Além dos efeitos lipogênicos, o hiperestrogenismo modifica o turnover das glicosaminoglicanas, com acúmulo de ácido hialurônico e condroitina-sulfato, aumentando a pressão osmótica intersticial, com a formação de microedema e dificultando a difusão de nutrientes, metabólitos e hormônios. Estes também provocam lassidão das paredes venosas na permeabilidade dos vasos linfáticos e alterações das arteríolas e capilares sanguíneos ${ }^{7}$.

A maior incidência de FEG ocorreu na região glútea (96,7\%) e na região posterior de coxa (90\%), provavelmente devido aos efeitos lipogênicos e hormonais já descritos, além de fatores ligados a hábitos físicos, 
nutricionais e emocionais ${ }^{8}$. O grau I esteve presente nas regiões anterior e medial da coxa, e os graus II e III apresentaram maior incidência nas regiões glútea, posterior e lateral da coxa. Houve também uma tendência à simetria, porém não exclusiva na região glútea e posterior da coxa.

Os adipócitos das regiões fêmoro-glúteas na forma ginoide têm maior volume e são dependentes dos hormônios sexuais femininos. São mais estáveis e resistentes à lipólise, por uma maior atividade da lipase lipoproteica e por existir um predomínio do efeito alfa-2-adrenoreceptor ${ }^{7,25}$.

Sandoval ${ }^{26}$ refere que a etiopatogenia do FEG também é relacionada a fatores mecânicos, tais como alterações posturais como pé plano, hiperlordose lombar, vestimentas demasiadamente apertadas e ortostatismos prolongado.

Piérard ${ }^{27}$ e Quatresooz et al. ${ }^{28}$ levantaram a hipótese de que nas áreas em que o FEG é mais evidente, a aparência da pele muda de acordo com a posição dos membros inferiores, em que a postura corporal tenderia a comprimir o tecido adiposo, acentuando as irregularidades cutâneas destas áreas específicas.

Milani, Natal Filho e Amado João ${ }^{29}$ sugerem que mudanças na curvatura da coluna lombar poderiam interferir com o suprimento sanguíneo local, influenciando a aparência e o grau do FEG. Para tentar confirmar esta hipótese, os mesmo autores realizaram um estudo prospectivo com 50 estudantes universitárias com FEG de coxas e glúteos, com idades entre 20 e 35 anos, e que não apresentavam sintomas de doenças neurológicas, respiratórias, circulatórias ou reumatológicas. Este tinha o objetivo de correlacionar o ângulo da lordose lombar com o grau do FEG, por meio de imagens radiográficas em perfil, analisando o ângulo de Cobb obtido pela intersecção das linhas perpendiculares ao nível do platô inferior da $12^{\mathrm{a}}$ vértebra torácica (T12) e do platô superior da $1^{\text {a }}$ vértebra sacral (S1). Não foi encontrada tal correlação, mas foram apontadas limitações do estudo tanto em relação ao método utilizado quanto à característica etiológica multifatorial do FEG, que torna complexa a eliminação de variáveis intervenientes, fatores que dificultaram a obtenção dos resultados, sugerindo a necessidade da utilização de outros métodos de avaliação postural, a fim de confirmar ou refutar a associação de alterações musculoesqueléticas e FEG.

Meyer et al..$^{14}$ selecionaram 30 mulheres com FEG e faixa etária entre 20 e 69 anos com o objetivo de desenvolver e aplicar um instrumento de coleta de dados, o qual permitisse avaliar de forma precisa o grau do FEG e os níveis das alterações sensitivas, possibilitando a otimização dos resultados obtidos tanto na avaliação quanto no tratamento a ser seguido. Todas as voluntárias apresentaram adiposidades localizadas, sendo 56,67\% ginoides, 33,33\% mistas e $10 \%$ androides. Para a determinação do FEG, foi utilizada a mesma classificação proposta no presente estudo ${ }^{9}$, na qual se observou que nenhuma das voluntárias apresentou exclusivamente o grau I, 80\% apresentaram os graus I e II e $20 \%$, os graus I, II e III. Esses dados corroboram com os do presente estudo, pois foram observadas as mesmas proporções de adiposidade localizada, sendo a maioria ginoide e a minoria androide; e em relação ao FEG, grande parte das mulheres foi classificada entre os graus I e II, uma menor quantidade entre os graus I, II e III associados e nenhuma apresenta exclusivamente o grau I.

Da amostra estudada, 100\% das voluntárias apresentaram FEG em alguma das regiões avaliadas. De acordo com a literatura ${ }^{4,13}$, a prevalência do FEG em mulheres jovens é de 85 a 98\%, esta acomete todas as raças, sendo mais comum nas caucasianas do que nas africanas. A região pélvica, os membros inferiores e o abdome são descritos como os locais mais comuns para o aparecimento do FEG ${ }^{1,3-5,9}$.

Devido a sua alta prevalência e seu consequente impacto na qualidade de vida, torna-se importante adotar estratégias de intervenções terapêuticas precoces por profissionais de saúde, de forma que o FEG deixe de ser tratado como uma disfunção exclusivamente estética, pois pode interferir na funcionalidade da mulher, assim devendo ser considerado e tratado como um problema de saúde ${ }^{14}$.

Portanto, concluiu-se que a região glútea e a posterior da coxa foram as mais acometidas e o grau I foi o mais incidente; e a porcentagem de gordura mostrou-se elevada nesta população com queixa de FEG.

\section{Referências}

1. Rossi AB, Vergnanini AL. Cellulite: a review. J Eur Acad Dermatol Venereol 2000;14(4):251-62.

2. Terranova F, Berardesca E, Maibach H. Cellulite: nature and aethiopathogenesis. Int J Cosmet Sci 2006;28(3):157-67.

3. Sadick N, Magro C. A study evaluating the safety and efficacy of the Velasmooth ${ }^{\text {TM }}$ system in the treatment of the cellulite. J Cosmet Laser Ther. 2007:9:15-20

4. Avram MM. Cellulite: a review of its physiology and treatment. J Cosmet Laser Ther 2004;6(4):181:5

5. Alster TS, Tanzi EL. Cellulite treatment using a novel combination radiofrequency, infrared light and mechanical tissue manipulation device. J Cosmet Laser Ther 2005;7(2):81-5.
6. Sant'Ana EMC, Marqueti RC, Leite VL. Fibro edema gelóide (celulite): fisiopatologia e tratamento com endermologia. Fisioter Especialidades 2007;30:5-1.

7. Ciporkin H, Paschoal LH. Atualização terapêutica e fisiopatogênica da lipodistrofia ginóide (LDG) “Celulite”. São Paulo: Santos, 1992.

8. Pires de Campos MSM. Fibro edema gelóide subcutâneo. Revista de Ciência \& Tecnologia 1992;77:82-1.

9. Rona C, Carrera M, Berardesca E. Testing anticellulite products. Int J Cosmet Sci 2006;28:169-73

10. Ulrich W. A celulite é curável. Rio de Janeiro: Tecnoprint, 1982.

11. Hexsel DM, Mazzucco R. Subcision: a treatment for cellulite. Int J Dermatol 


\section{$2000 ; 39: 539-44$}

12. Guirro ECO, Guirro RRJ. Fisioterapia Dermato-funcional: fundamentos, recursos e patologias. São Paulo: Manole, 2002.

13. Velasco MV, Tano CT, Machado-Santelli GM, Consiglieri VO, Kaneko TM, Baby AR. Effects of caffeine and siloxanetriol alginate caffeine, as anticellulite agents, on fatty tissue: histological evaluation. $\mathrm{J}$ Cosmet Dermatol 2008;7(1):23-9.

14. Meyer PF, Lisboa FL, Alves MCR, Avelino MB. Desenvolvimento e aplicação de um protocolo de avaliação fisioterapêutica em pacientes com fibro edema gelóide. Fisioter Mov 2005;18(1):75-83.

15. Neto MF, Francischelli RT, Oliveira AP. Estudo da composição corporal e suas implicações no tratamento da hidrolipodistrofia e da síndrome da desarmonia corporal. Rev Soc Bras Med Est 2003;20:7-15.

16. WHO, Global Database on Body Mass Index [database on the Internet]. Disponível em URL: http://www.who.int/bmi/index.jsp?intro Page=intro_3. html. Acessado em 23 de maio de 2008.

17. Salem M, Fernandes Filho J, Pires Neto CS. Desenvolvimento e validação de equações antropométricas específicas para a determinação da densidade corporal de mulheres militares do Exército Brasileiro. Rev Bras Med Esporte 2004:10:141-6.

18. McArdle WD, Katch FI, Katch VL. Fisiologia do exercício: energia, nutrição e desempenho humano. Rio de Janeiro: Guanabara Koogan, 2003.

19. Heyward VH. Avaliação física e prescrição de exercícios: técnicas avançadas. Porto Alegre: Artmed, 2004.

20. Pitanga FJG. Testes, medidas e avaliação em Educação Física e esportes. São Paulo: Phorte, 2004.
21. Querleux B, Cornillon C, Jolivet 0 , Bittoun J. Anatomy and physiology of subcutaneous adipose tissue by in vivo magnetic resonance imaging and spectroscopy: relationships with sex and presence of cellulite. Skin Res Technol 2002;8(2):118-24.

22. Mirrashed F, Sharp JC, Krause V, Morgan J, Tomanek B. Pilot study of dermal and subcutaneous fat structures by MRI in individuals who differ in gender, BMI, and cellulite grading. Skin Res Technol 2004;10(3):161-8.

23. Ortonne JP, Zartarian M, Verschoore M, Queille-Roussel C, Duteil L. Cellulite and skin ageing: is there any interaction? J Eur Acad Dermatol Venereol 2008;22(7):827-34.

24. Rosenbaum M, Prieto V, Hellmer J, Boschmann M, Krueger J, Leibel RL, et al. An exploratory investigation of the morphology and biochemistry of cellulite. Plastic Reconstructive Surg 1998; 101 (7):1934-9.

25. Rotunda AM, Suzuki H, Moy RL, Kolodney MS. Detergent effects of sodium deoxycholate are a major feature of an injectable phosphatidylcholine formulation used for localized fat dissolution. Dermatol Surg 2004;30(7): 1001-8.

26. Sandoval B. Fibroedema gelóide subcutáneo: qué conocemos de esta entidad clínica? Folia Dermatol 2003;14:38-42.

27. Piérard GE. Commentary on cellulite: skin mechanobiology and the waistto-hip radio. J Cosmet Dermatol 2005;4(3):151-2.

28. Quatresooz P, Xhauflaire-Uhoda E, Piérard-Franchimont C, Piérard GE. Cellulite histopathology and related mechanobiology. Int J Cosmet Sci 2006;28(3):207-10.

29. Milani GB, Natal Filho A, Amado João SM. Correlation between lumbar lordosis angle and degree of gynoid lipodystrophy (cellulite) in asymptomatic women. Clinics (Sao Paulo) 2008;63(4):503-8. 